\title{
Buch-Anzeigen
}

Handbueh der Gynäkolog·ie. Herausgegeben von J. Veit. Bd. 1: Löh-lein, Antisepsis und Asepsis: Kiistner, Die Lage- und Bewegungs-anomalieen des Uterus und seiner Nachbarorgane. Besprochen von Werth eim -Wien.

Asepsis und Antisepsis. Yon 11. Löhlein in Giessen. Die Arbeit gliedert sicli in 3Abschnitte; der erste ist der subjectiven Asepsis, der zwoito der Sterilisation der Instruniente, des Näh- und Yerbandmateriales, der dritte der Vorbereitung der Kranken gewidmet. Für die Erzielung subjectiver Asepsis stellt L. folgende 3 Grundsätze auf: 1. Man habe die Möglichkeit, sich, seine Hände, Kleider und Instruniente zum Träger pathogener Keime werden zu lassen, zu ve $\pi$ neiden. 2. Unmittelbar nach trotzdem s tattgehabter Yerunr ein i gun $g$ habe man eine gründliche Waschung und Desinfection vorzunehmen. 3. Yor jeder Operation und vor jeder Untersuchung habe man zu überlogen, ob cine Gelegenheit zur Yerunreinigung vorausgegangen.

Trotz der Aufstellung dieser Forderungen halt L. eine Abstinenz im Allgemeinen für nicht nöthig. Nur in jenen Fallen, in denen eine völlig genügende Desinfektion durch Hyperästhesie, durch eczematöse, furunkulöse und ähnliche Prozesse an den Händen und $\Lambda^{\wedge}$ orderarmen unmöglich gemacht sei, oder $\Lambda$ venn seit dem Momente einer lnfektionsgelegenlieit nicht genügend lange Zeit verstrichen, um drei- bis viermal $\Lambda$ viederholte Desinfektion der exponirten Körpertheile auszuführen, ein Vollbad zu nehmen, Wäsche und Kleider vollständig zu wechseln, habe man Abstinenz zu lieobachten.

Nach einer Auseinandersetzung über die gebräuchlichsten chemischen Desinfizientien wird die Desinfektion der Hände besprochen. Wenn auch die mechaiiische Reinigung der Hände an und für sich nicht ausreiche, so habe doch jede Desinfektion mit einer möglichst gründlichen meehanischen Reinigung mit Seife und Wasser zu beginnen; der P†lege der Haut und der Nägel, speziell der Unternagelräunie und der Nagelfalzo, habe man besondere A.ufinerksamkeit zuzuwenden. Von grosser Bedeutung sei es, dass die $\lambda$ Vaschungen mit möglichst warm em Wasser vorgenommen werden, indent hierdurch aller Schmutz schneller und gründlicher gelöst und weggeschafft

Buchanzeigen. 271

werde, als durcli kaltes Wasser. Der von Ahlfeld geforderten ,Intensität” der Waschung raisst auch L. grossen Wertli bei, eine rein mochanische Des-ínfection halt er aber nicht für ausreichend.

Bei der Besprechung der chemischen Desinfektion der Hände ver-breitet sich L. hauptsächlich über die nenereii Àrbeiten Ah If eld's, Ileinecke's, Pot en's und Krönig's über den Worth des Alkohols und betont, dass eine Einigung dor differenten Ansichten bisher nicht erzielt sei. Er anerkennt don Worth der Neuprüfung unsorer Händedesinfection auf bacteriologisehem Wege, warnt aber, zu vorgossen, dass dor Begriff dor Keimfreiheit im bacteriologischen Sinne sich mil deni dor genügenden aseptischen Vorbereitung im klinischen Sinne nicht decke. Die höchste und letzte Entscheidung über den Werth der Desinfektionsmethoden werde durch $<$ lio klinische Erfahrung gegeben. 
Im zweiten Abschnitte wird nach einer Besprechung dor verschiedenen Sterilisationsmetboden für die Instrumente und dor aseptischen Anwendung von Specula, Uterussonde, Curette, Katheter und der verschiedenen Quell-mittel zur Dilatation des Cervixkanales der CatgutSterilisation besondors eingehendo, Beaclitung gewidmet. Bei dem Umstande, als in dieser Frage noch fortwährend neue Versuche unternommen werden, und dass bisher noch keine einzige wirklich einwandsfreie Methode der Catgut-Sterilisation be-kannt ist, ist os von Interesse, zu erwähnen, dass L·öhlein für dieselbe die trockene Hitze anwendet und mit den hierbei erzielten $1 \cdot$ iesultaten vollständig zufrieden ist.

In dem Capitel „Operationsraum” he,bt L. hervor, dass bei sorgfältiger Anwendung der aseptischen und antiseptischen Vorschriften auch in modernen $\Lambda$ nforderungon nicht entsprechenden Lokalitäten gute Resultate erzielt werden können.

Was die Vorbereitung tier Kranken für die abdomincllen und vagina] on Operationen betrifft, welche in dem dritten Abschnitte ilire Besprechung findet, fordert L. gründliche Entleerung des Darmes und sorgfältige Desinfektion. Besonders gründlich habe $<$ lio lokale Desinfektion bei vaginalen Operationen zu sein; die Vagina sei wegen dor vielen Buchten und Falten ihrer Wandung am besten so zu desinfiziren, dass sic mit don Inngern gründlich ausgewischt wird. Bestehen furunculöse odor eczematöse Prozesse, clami müssten dieselben vorerst zur Ausheilung gebracht werden.

Was die Asepsis und die Antisepsis beim Operiren selbst betrifft, werden die grossen Vorzüge der ersteren entsprechend gewürdigt. Trockene und feuchte Asepsis werden in ihrem Werth e verglichen: Löhlein ist ersterer treu geblieben.

In Bezug auf Eitererguss bei peritonealen Operationen wird der Werth der Drainage besprochen; L. erwähnt hierbei, dass er bisher in alien Fallen mit Eitererguss in die Banchhöhle Drainage angewendot habe, und zwar nach dor Methode von Mikulicz.

Die Ueberlegenheit des aseptischen über das antisept - ische Verfahren illustrirt L. durch die geradezu glänzenden Resultate, die or im Laufe dor letzten 11 Monate in der Giessener Frauenklinik erzielt hat: von 75 Lapa-ratomirten sind nur 3 gestorben, jedoch keine an den Folgen dos Eingriffes.

Indem sich L. dahin ausspricht, dass, ebonso wie die Opfer, welche die grossen Entbindungsanstalten chedem forderten, auch die septischen Er-krankungen nach don grossen gynäkologischen Operationen in absehbarer Zeit der Geschichte angehören $\Lambda$ verdon. schliesst die Abhandlung.

$\mathrm{L}$ a g e - und B e w e g u n g s a n o m a 1 i e n des U t e r u s u n d seiner $\mathrm{N}$ a c h -barorgane. Von Otto Küstner in Breslau.

DӨï erste Abschnitt handelt von den normalen Lagen und Bewagungen dos Uterus und enthält eine Darstellung der Anatomie des Ligamentapparates auf Grund dor Mackenrodt'schen und Ziegenspeck'schen Üntersuchungen. I in zweiten Abschnitte, „Die Anomalien der Beweglichkeit dos Uterus", finden die pathologische Fixation und die abnorme Beweglichkeit dos Uterus Darstellung. K. halt es für angezeigt, die Anteversio-flexio aus den Begriffen, welche Pathologisches bezeichnen, zu streichen. Nach ihin giebt es keiue pathologische Anteversioflexio, und or schlägt vor, für diejenigen pathologi-

272 Buchanzeigen.

schen Fiille für welche Schultze den Ausdruck Anteversio oder -flexio bei-behalten habe, lieber den Ausdruck patholo gis che Fixation des ante-vertirten respective -fleetirten Uterus zu $\Lambda$ vählen. 
Selir bemerkenswerth ist, dass K. in Bezug auf die Parametritis posterior, welclie das grösste Kontingent zu den S c hul tz e'sehen pathologischen Ante-versionen-flexionen stellt, der Ansicht ist, dass es sieh hierbei meistens nicht $\pi \mathrm{m}$ parametritische, sondern urn chronische perimetritisclie Prozesso handle.

In dem Absehnitte íiber die pathologischen Lagen des Uterus und seiner Nachbarorgane werden kapitelweise Elevation, Ante-position, Retro-position, Lateralposition, Lateralversion-flexion, Torsion nach ihrer klinischen und anatomischen Dignität eingehend gewürdigt und die mannig $1 / 8$ chen Ur-sachen, welche diese Zustände herbeiführen, aufgeführt.

Retroversio-flexio und Descensus hat K. wegen ihrer innigen Be-ziehungen in einem Kapitel gemeinsam behandelt. Was die Aetiologie der Retroversio-flexio betrifft. so erweitert K. das von Schultze angegebeno Schema, indem er als cine selir wichtige und häufige Ursache die Pelveoperitonitis hinzufügt. Audi halt er es in Uebereinstimnnmg mit seinen früheren Untersuchungen für wahrscheinlich, dass zu lange und consequent eingehaltene Rücke@lage im Puerperium das Entstehen der fehlerhaften L·ago unterstütze.

Bei Besprechung der pathologisch-anatomischen Yeränderungen, die sich bei Retroversio-flexio im Uterus und seinen Nachbarorganen finden, spricht sich K. dahin aits, dass, wenn aueli zugegeben werden müsse, dass die so oft vorhandene und zur Fixation des Uterus führende Entzündung des Perimetriums ihre Ursache sehr oft in mikrobiotischen Tnfectionen habe, dieselbe in einer Reihe von Fallen als eine Folge der abnormen Lagc auf-gefasst werden müsse. Die Retroversio-flexio sei gleichbedeutend mit ab-soluter Ruhelage (?), die norm ale Mechanik der Beckenorgane sei lahmgelegt, und daher erleiden die gegenüberliegendeu, einander dauernd berührenden Partien der serösen Flächen Drucknekrose des Endvtliels, wodurch eine Yerlõthung entstehc.

In Bezug auf die Therapie der Retroversio-flexio wird die bima-nuelle Reposition ausführlicli besprochen. Dass das Küstner'sche Ver-fahren hierbei Vorzügliches leistet, hat Ret', oftmals praktisch erfahren. Der rectovaginale Handgriff (Zeigetinger im Rectum, Daumen im vorderen Schei-dengewölbe) wird nicht erwähnt.

K. halt das Schultze'sche Verfahren, in tiefer Narkose in einer Sitzung die Lösung einer fixirten Retroversio-flexio zu erstreben, für un-vergleichlich rationeller als die Massage, ein $\Lambda$ ussprnch, dem sich Rof. nach seinen Erfahrungen unbedingt anschliessen muss. Es ist kein Zweifel, dass in jenen Fallen, in welchen Aufrichtungsvei suche in tiefer Narkose bei fixirter Retroversio-flexio nicht zum Ziele gefñhrt haben, die Massage vollständig erfolglos bleib-t, wälirend umgekehrt in vielen Fallen, in denen lange Zeit fortgesetzte Massage nicht zum Ziele führte, das Schultze'sche Verfahren von Erfolg begleitet war.

Die Mittel, urn den aufgerichteten Uterus dauernd in der richtigen Lage zu erhalten, werden hierauf besprochen. Bezüglieh der Pessare steht K. auf dem Standpunkte, dass, so mannigfach die vorhandenen Formen seien, in $\Lambda$ Virklichkoit die Schult ze'sche 8, das Smith'sche und das Tliomas-sche Pessar genügen. Die Wirkungsweise jeder dieser Formen wird aus-führlich erörtert und als Yortheil des Schultze'sehen Pessars hervor-gehoben, dass es Individualisirung gestatte.

Modifikationen erfahre der Gang der Behandlung durch die Berück-sichtigung von Ivompl·ikationen respective Fulgezuständen, welclie bis zu einem gewissen Grade zu den typischen gehören: Endometritis und Cervix-und Dammdefecte. Für die Erstere wird als am schnellsten zum Ziele führend die Ausschabung empfohlen, für die Letztere der plastischoperative Weg. Am besten sei es, in solchen Fallen auch gleich auf die übliche orthopädische Correctur der falschen Lage mittelst Reposition und Pessar zu verzichten und dieselbe durch eine Retroflexionsuperation zu ersetzen. 


\section{Buchanzeigen. 273}

Was die operative 1/8angriffnahme der Retroversio-flexio betrifft, so wird die Entwicklung und die spezielle Teehnik der verschiedenen Ver-t'aliren auseinandergesetzt: die Operation Alexander's, Ols ha us en's und Dülirssen-Mackenrodt's. Für das leistungsfähigste Verfahren halt K. die Àlexander'sche Operation. Die neue Operation: die Verkürzung der L·ig. rot. mittelst des vorderen Scheidenbauchschnittes, vom Referenten und von Bode angegeben, erwähnt K. noch nicht. Mit einer kurzen.Zusammen-fassung des therapeutischen Yorgehens in Fallen von Retroversio-flexio schliesst das nach jeder Richtung hin erschöpfendo Kapitel über Retroversioflexio.

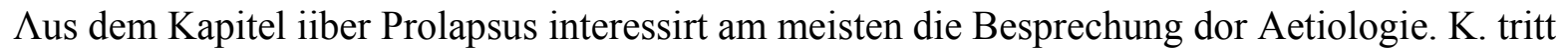
der verbreiteten Anschauung, dass mangelhafte puerperale Involution der Genitalien, spezioll der Scheide, die primäre Ursache des Vorfalls sei, entgegen. Nach seiner Anslcht entstehen die Prolapse in der Regel aus Retroversio-flexionen: diese Lageveränderung sei das Vorstadium des Prolapsus, und demgemäss seien die Entstehungsbedingungen des Prolapsus identisch mit denen der Retroversio-flexio. Die Bcdingungen aber, unter welchen aus der Retroversio-flexio der Prolaps entstehe, seien vielleicht in vereinzelten Fallen gegeben durch ganz besondere Schlaffheit der Stützapparate des Uterus: in der Hauptsache aber seien die statischen Yerhältnisse bei Retroversio-flexio schuld: der kunstante intraabdominale Druck treibe den retrovertirten-flectirten Uterus immer weiter nach abwär†łs, und indem sich frühzeitig zur Erschlaffung und Faltenbildung der vorderon Scheidenwand eino Cystocele hinzugeselle, so trete als weiteres schädigendes, den Prolaps förderndes Moment hinzu, dass vvegen der Dislokation der Blase die Entleerung dieses Organes Schwierigkeiten zu machen beginne. Da infolge (lessen die Bauclipresse starker in Thätigkeit gesetzt werden müsse, werde das prolabirte Stuck vorderer Scheidenwand immer weiter nach aussen gedrängt, und so komme theils (lurch den Zug dieses, theils durch den Druck dor Bauclipresse von vben eine Vervollständigung des Vorfalles zu Stande. Die nun hinzutretenden Stauungserscheinungon ergänzen das Bild. Dies ist nach K. der gewöhnliche Mechanismus beim Zustandekommen eines Prolapses: den primären Prolaps der vorderen Vaginalwand halt er allerdings für möglich, aber für den bei $\Lambda$ veitem selteneren. Noch seltener sei die Entstehung des Prolapses durch primäre Hypertrophie der Portio vag. (Col tapyroide).

Was die Therapie betrifft, hatKüstner von der Massage nach Thure Brandt thatsächliche Erfolge gesehen. Er bezieht dieselben aber vveniger auJ" tlie Anregung der Ligamentmuskulatur und auf einen vortheilhaften Ein-fluss auf die ödematös-hypertrophischen Cervicalpartien als vielmehr auf die Besserung respektive Beseitigung der beim Prolaps so häufigen und auch ursächlichen Perimetritis adhaesiva. Da die Periinctritis Retroversio-flexio verursachen $\mathrm{k}<$ "mne, so könne sie auch die Ursache des Prolapses sein. $\Gamma$ n diesen Fallen könne die Ligamentmuskulatur lange Zeit leistungsfähig bleiben und dem Uterus nach Beseitigung der perimetritischen Adhäsionen wieder die Normallage gewährleisten.

Imnierhin sei es nur ein geringer Bruchtheil von Prolapsen, welcher durch die Massage geheilt werden könne.

Was die Pessartherapie betreffe, so liege auch Hire Hauptaufgabe in der Korrektur der den Prolaps begleitenden resp. verursacht habenden Retroversio-flexio. Für am meisten bewährt erklärt Küstner demgemäss dieselben Pessare, die für die Retroversiû-flexio erprobt seien. Nur wenn die Scheidenschleimhaut mager, brüchig sei und daher einen stärkeren isolirten Druck nicht vertrage, $\mathrm{o}<$ ler wenn die Form der Scheide eine konische sei, seien diese Formen nicht angezeigt. Hier müsse man auf die Rechtlagerung des Uterus verzichten und sich damit 
begnügen, ohne Rücksicht auf die Stellung des Uterus die Reposition des Sclieidenvorfalls anzustreben. Unter diesen Ümständen leisten die Eipessare nach Breisky, die, Schalenpessare und andere Formen ltessere Dienste.

Auch die verschiedenen Methoden der Colpurrhaphia anterior und posterior und der Perineorrhaphie linden eingehende Besprechung. (Ebenso

\section{Buchanzeigen.}

$\Lambda$ vie Baum hat aiu 'li die Klinik Schauta's mit der b1 reund'schen Methode der subkutanen etagenweisen Anlegung von Silberdrahtringen nur ungün-stige Erfolge aufzuweisen gehabt. Wenn aacb der momentane Effekt ein ausgezeichneter ist, so kommt es sehr rasch infolge des Durehschneidens der Drãlite zu neuerlichem Vorfall.)

Aber keine von den Operationen, bei denen es sich im Wesontlichen nur um die Verengerung der Scheide handle, könne wirklich befriedigen, wei] dieselben die gest $<$ " $>$ rte Mechanik der inneren Genitalien, vor Allem die fehlerhafte Lage des Uterus, niclit berücksichtigen, woraus sich die so häu-figen Rezidiven nach diesen Operationen erklären. Deswegen hat Küstner schon im Jahre 1888 mit den scheidenverengernden Operationen die Ventri-fixation verbnnden. und die damit erzielten Result-ate waren für ihn so er-muthigend, dass er späterhin fast ausschliesslich so vorging.

Auch darauf kommt K. zu sprechen. dass man die Ventrifixation er-setzen könne durch andere operative Verfahren (Alexander-Adams, $\Lambda$ 7aginofixation etc.); nur niüsse dann vorher das Cervixoedem zum Ab-schwellen gebracht worden, oder es müsse die Cervixaniputation vorgenommen werden: was bei Ausführung der Ventrifixation überflüssig sei.

1111 12. Kapitel wird Inversion und Eversion behandelt. Nach Darstellung der Anatomic, Aetiologie, Symptome und Diagnose der puer-peralen und der durch Tumoren bedingten Inversionon wird bezüglich der ïherapie liervorgehoben, dass bei frisch entstandenen puerperalen Fallen die Reïnversion leicht gelingt, dass aber jeder Tag des Abwartens infolge zu-nehmender Verengerung des Inversionstrichters die Schwierigkeiten ver-grössert, die Gefahren steigert. $\Lambda$ Vährend bei fríschen Fallen das rein manuelle Verfahren wohl immer ausreiche, sei bei den veralteten Formen die Anwendung des Colpeurynters das Zweckmässigste. Führe die Colpeurvse niclit zum Ziele, so müsse der operative $\Lambda$ Veg eingesclilagen werden. Es ist ein grosses Verdienst Küstner's, dass er an Stelle der verstüm-melnden Operationen (Amputation und Exstirpation des Uterus) cine prak-tisclie und relativ leicht durchzuführende operative Methode der Reïnversion gesetzt hat. Dieselbe besteht im Wesentlichen aus einer von der Scheide her vorzunehmenden blutigen Dilatation des Inversionstrichters. Küstner erõffnef den Douglas'schen Raum von der Scheide aus, spaltet hierauf die hintere Uteruswand genau in der Meridianlinie durch einen longitudinalen Schnitt, der etwa ' $2 \mathrm{~cm}$ uber dem äusseren Muttermund anfängt und ' $2 \mathrm{~cm}$ unterhalb des Fundus endigt: hierauf gelingt die Reïnversion spielend. Indem nun das künstlich stark retroflectirte corpus uteri (lurch das hintere Scheidengewölhe in die Scheide hereingezogen wird, wird der gesetzte Medianschnitt von der Peritonealseite her der Naht zugänglich. Der ge-nähte Uterus wird reponirt, hierauf die Wunde im Douglas verschlossen. Mit Recht hebt K. hervor, dass dieses Verfahren, das er ýbrigens schon im Jahre 1893 angegeben hat, berufen sein dürfte, die Coeliotomie einerseits, die verstümmelnden Operationen andererseits vollstándig zu verdrängen.

Bezüglich der durch Tumoren bedingten Inversion schliesst sich $1 \backslash$. der Ansiclit von Schauta an, dass auch diese Form der Inversion nur während eines Erschlaffungsstadiums des Organes zu Stande konimen kann. Die, Schwierigkeit der Differentialdiagnose gegenüber einfach gestielten Myomen wird liervorgehoben, und als goldene Regel empfohlen, alle in die Scheide geborenen 
Myoine nach dem Typus der Enucleation zu entfernen. Gelinge die Re $1 / 8$ version nach Entfernung des Tumors niclit, dann liabe man sich zur Amputation oder Totalexstirpation zu entschliessen. Das letzte Kapitel behandelt die Hernia uteri, Hysterocele. Bezüglich der Entstehung derselben weist Küstner auf die Ansicht Schultze's hin, $\Lambda$ velcher angeborene Kürze des Uigamentum rotundum als ätiologisches Moment erwähnt hat.

Lehrbueh der Naehbehandlung · naeh Operationen. Von Paul Reichel.

Vorlesungen fur Studierende und Aerzte. Wiesbaden. J. F. Bergman n. 181)7.

Verfasser hat sich mit sehr gmssem Geschick und grosser Gründlich-keit dei· Aufgabe

unterzogen, des Genaueren die Art der Nachbehandlung

Buchanzeigen. 275

nach den einzelnen Operationen zu schildern mid dem angehenden Arzto mid Chirurgen eine Menge von Lehren und praktischen $\Lambda$ Vinken zu geben, die or vergebens in unseren Lehrbüchem der Chirurgie suchen wird. Aber auch dor ältere Chirurg wird in dem fliessend geschriebenen Buch eine Menge $\Lambda$ Vissenswertes finden und nicht ungern die einmal begonnende Lecture fortsetzen. Der Inlialt ist in 32 Vorlesungen verteilt (463 Seiten), von denen 11 sich auf die allgemeine Nachbehandlung nach Operationen, 21 auf die Nachbehandlung nach Operationen an den verschicdenen Organen beziehen. Wenn auch der allgemeine Toil der Hauptsache nach nur spociell chirurgi-sches Intoresse bietet, so ist doch das über die Anwendung der Drainage, Tamponade der Wunden, Desinfection, Störungen des Wundverlaufs, $\Lambda$ Vundinfectionskrankheiten Gesagte bei der Gründlichkeit und Objectivität des Verfassers auch don angehenden Gynäkologen als Lecture warm.zu empfohlen. Ini specicllen Teil interessirt in gynäkologischer Beziehung besonders die Nachbehandlung nach Coeliotomien, deren Besprechung zwei Vorlesungon gewidmet sind. Verf. erachtet mit Recht bei normalem Verlauf nach asep-tischen Coeliotomien Opium als zwecklos, zu $\Lambda$ veilen als schädlich. Zur Ver-hütung eines Bauchbruches sollen die Operirten, wenn möglich, erst nach der 3. $\Lambda$ Voche aufstehen und dann jioch fur mehrero Monate eine gut sitzende Bandage tragen. Eingehend werden fernor die Symptome der Shocks, der inneren Blutung, der Inanition, der vorschiedenen Formen der peritonealen Infection, des Ileus und Pseudoileus besprochen, ihre Differentialdiagnose beleuehtet und sehr beherzigenswerte Winke für unser therapeutisches Han-deln gegeben. Es ist dieses Capitel eines der interessantesten des ganzen Buchos, besonders deshalb, $\Lambda$ veil Verfasser sich in früheren. Arbeiton spocioller mit diesem Gebiete beschäftigt hatto. Bei dor Behandlung $\tau ı m$ nicht ex-stirpirbaren Oysten, die mit dor Bauchwunde vernäht werden mussten - Cysten des Mescnterium, der Milz, der Leber empfiehlt R. für die ersten 14 Tago die Tamponade mit Craze. Der Vollständigkeit $\Lambda$ vegen hat der Verf. eine Vorlesung der Nachbehandlung nach Operationen an don weiblichen Genitalien gewidmet.

Wenn auch dor diesem Capitel gewidmete Raum nicht sehr gross ist, so sind doch die Grundzüge klar dargelegt. Einzelheiten werden weniger be-ri'icksichtigt wie in den anderen Capiteln.

Besonders hobt R. die $\Lambda$ Vichtig-keit einer sehr peinlichen Desinfection bei alien

Fisteloperationen und Plastiken hervor. Bei letzteren ist er ein Gegner der künstliclien Olistipation. Nach alien intrauterinen Operationen bevorzugt er die Jodoform-gazetamponade; die antiseptischen Ausspülungen vorwirft er, sofern es sich nicht nm eine Retentio in cavo uteri handelt. Nach vaginaler Hysterektomie soil, wenn möglich, das Peritoneum durch die Naht geschlossen werden. Dass bei lvlammerbehandlung die Blutung nach 2 Tagen sichor steht, wie Verfasser moint. ist wohl nicht immer der Fall. 
1 'js ist nicht möglich, ohne den Rahmen eines líefera†es zu über-schreiten. noch näher auf die Einzelheiten dioser Vorlesung eiuzugehen. Es ist schade, dass einzelne Abschnitte derselben wie dio Salpingotomie, Ventro-fixation, Myvmotomie nicht ausführlicher behandelt worden sind. Das Erscheinon des Lehrbuclies kann nur mit Freuden begrüsst werden und seine Lecture kann Jedem, dor sich auf diesem Gel > iet genau informiren will, sehr warm empfohlen werden. B orch ard -Posen.

Baetepiologie des weiblichen Genitalkanals. Von C. Menge und B.

Krönig, Privatdvcenten und Assistenten an der Eiiiversitiits-Frauenklinik in Leipzig. 2 Theile. Leipzig., A. Georgi. 1897. I. Theil: Menge, Bakteriologie des Gen it a Ik an ales der nicht s ch w anger en und nicht puerperalen Fran.

Die bei der Besprechung von Biichern häutig sich wiederholende Be-nierkuiig, dass das betreffende Work oinem Bedürfníss entspreche und eine Lücke ausfülle, gilt in seltenem Masse für das vorliegende Menge-Krö-nig"scho $\Lambda$ Verk. Denn gerade auf dem Gebiete dor Bakteriologie dor weiblichen Genitalorgane existiron so violo, den Anspruch auf Glaubwürdigkcit erhebendo, sich so völlig widersprechende Beobachtungen und Enter276 Buchanzeigen.

suchungen, dass eine erschöpfende litterarische Behandlung and vor Allem eine streng wissenschaftlíche Nachprüfung von Jedem, der sich fur diese Fragen interessirt, schon längst als ein dringendes Bedürfniss empfunden worden ist. Bislang konnte ein Jeder so ziemlich jede überhaupt mögliche Behauptung iiber Bakterienbefunde ini weiblichen Sexualapparat aufstellen und sich dabei auf Namen von sonst gutem Klange beruien. Die Unter-suchungsergebnisse von Winter, Steffeck, Witte, "Walthard, Stro-g an off, Maslowsky, Samschin, Doederíein und der beiden Verfasser (von zahlreichen sonstigen Arbeiten ganz zu schweigen) waren so widerspruchsvolle, dass nur ein ganz intimes Eindringen in die Untersuchungs-methoden der verschiedenen Forscher einen Fingerzeig dafür abgab, auf welcher Seite die Untersuchnngsfehler und auf weícher die einwandsfreiere Methode zu finden sei. $\Lambda$ Ver allerdings die zahlreichen Arbeiten, die die beiden Verfasser im letzten halben Jahrzehnt über diesen Gegenstand veröffentlicht haben, aufmerksam verfolgt hat, war über das schliessliche Er-gebniss ihrer Untersuchungen nicht mehr im Unklaren; um so befriedigter wird er sein, dass die Verfasser die langwierigsten und mühseligsten Untersuchungen nicht gescheut haben, um einen wirklich überzeugenden Nachweis †ur die Richtigkeit ihrer Behauptungen zu erbringen und um die Wider-sprüche seitens anderer Autoren aufzuklilren oder zu besiegen.

Nach unserer Meinung ist durch das vorliegende $\Lambda$ Verk eine grosse Reihe bis dahin umstrittener Fragen für längere Zeit zum endgültigen $\mathrm{Ab}$-schluss gebracht.

Wie aus der Ueberschrift hervorgeht, hat sich Menge die Aufgabe gestellt, die nicht schwangeren Genitalien auf ihren Bakteriengehalt zu untersuchen, indem er Krönig die Erforschung der schwangeren Genitalien überliess.

Als Nährböden verwandte er 1. schwachalkalisch.es Agar, 2. schwachsauer reagierendes Agar. 3. Traubenzucker-Agar (alkalisch), 4. Glycerin-Agar (al-kalisch), $\cdot \Gamma>$. u. ( $>$. alkalisch und sauer reagirende Gelatine, 7. Traubenzucker-Gelatine, 8. Bouillon, í). Traubenzucker-Bouillon. Der Sinn dieser verschiedenen Nährböden liegt darin, dass der Verf. nicht nur Pilze, die auf gewöhnlichen alkalischen Nährböden wachsen, sondern auch solche kultiviren wollte, die nur auf sauren Nährböden gedeihen. Der Traubenzuckerzusatz geschah stets zu hoch geschichteten Culturgläsern; der reducirend wirkende Zucker erlaubt auch den obligaten Anaëroben das Aufkeimen. Das Glycerin-Agar ermöglichte das Angehen der Tuberkelbacillen; dasselbe wurde ver-mischt init Kystomflüssigkeit im Verhältniss von 2 : 1. Zur Züchtung der Gonokokken $\Lambda$ - 
erwandte er schliesslich eine Mischung von schwacb alkalischem Agar ebenfalls mit Kystomflüssigkeit.

$\Lambda$ Venn wir jetzt zur Besprechung der einzelnen Kapitel übergehen, so handelt das erste Kapitel von den Baktorien der gesunden und kranken Vulva, Urethra, Glandula Bartholini.

Während Menge im Kindesalter eine durch den Gonokokkus bedingte $\Lambda \Gamma u$ uvitis anerkennt, leugnet er dieselbe bei Er $\Lambda$ vachsenen. Allerdings finde man häufig bei frischer Gonorrhoe eine Yulvitis und könne hier auch die Gonokokken leicht nachweisen. Die Thatsache jedoch, dass diese Vulvitis durch einfaches Säubcrn und Trockenhalten der äusseren Geschlechtstheile in kürzester Zeit zur iiieilung kommt, ohne dass die Gonorrhoe im Uebrigen sich bessert, beweise, dass der Gonokokkus nicht der Erreger der Vulvitis sei, sondern, dass letztere durch das von oben herabfliessen.de Sekret ent-standen sei.

Ebenso sei auch die Vaginitis Erwachsener gemeinhin nicht als gonorrhoisch zu bezeichnen. Den Beweis dafür sieht M. in der Thatsache, dass in dem Scheidensekret Gonorrhoischer ausser dem Gonokokkus sich stets zahllose andere Bakterien nachweisen lassen. Menge halt es aber (in võlliger Uebereinstimmung mit $\Lambda$ Vertheim) t'ür ausgemacht, dass der Gonokokkus eine Symbiose mit anderen Keimen nur höchst ungern eingeht. Der Befund der Gonokokken in der Scheide stamme vielmehr aus dem Cervical-sekret; von. hier aus werden die Gonokokken in die Scheide geschwemmt, ohne hier festen Fuss fassen zu können. Er kommt im nächsten Capitel auf diese $\Lambda$ rerhältnisse noch ausführlicher zurück.

Buchanzeígen.

277

Die Urethritis ist nach Menge meistens gonorrhoischen Ursprungs. Er weicht hier etwas von dor Behauptung Bnmm s ab, welcher sagt, dass er keine Urethritis je gesehen habe, die nicht gonorrhoisch sei. Menge hat unter 50 bakteriologisch verarbeiteten Uretbritiden 2 Fälle, in denen sich mikroskopisch wenigstens keine Gonokokken nachweisen liessen. Beides waren acute P'älle bei älteren Frauen, welche in kürzester Zeit geheilt waren.

Die Bartholinitis ist in der Mehrzahl der Fälle gonorrhoischen Ursprunges. Meist findet man im Eiter Gonococcen, dagegen nur selten den Staphylococcus pyogenes aureus, nicht allzu selten ist der Eiter steril.

Das zweite Capitel handelt vom Keimgehalt der Vagina und mufasst die interessantesten und am heissesten umstritteneu Gebiete der ganzon Frage. Die Wichtigkeit dieser Frage liegt allerdings nicht in dem Theïl der Arbeit, deren Erledigung sich Menge zur Aufgabe gestellt hat, als vielmehr in dem Krönig'schen Antheil: 1st die schwangere Scheide frei von In-fectionserregern, giebt es eine Selbstinfektion und sind präliminare Scheiden-douchen vor der Geburt erforderlich? Gegenüber dicsen Fragen treten zwar alle iïbrigen an Bedeutung weit zuriick; jedoch lassen diese sich nur ver-stehen und auch nur begründen, wenn die bakteriellen Verhältnisse der nicht schwangeren Scheide erforscht und klargestellt sind. Gerade die bisherigen Untersuchungen über den Keimgehalt der Scheide, sowohl der schwangeren vie der nicht schwangeren, wiesen so auffällige Widersprüche auf, dass - wie in der Einleitung gesagt wurde - so ziemlich jede Angabe sich durch scheinbar zuverlässige Beobachtungen begründen liess.

Der Grund der $\Lambda$ rerschiedenartigkeit der bisherigen Ergebnisse lag in einer ungenügenden Methode. Da es von geringem Interesse ist, zu er-fahren, was für Keime in die Vagina hineingel angen können, es viel-mehr $\Lambda$ vesentlich darauf ankommt, welche Keime in der Vagina leben können, so benutzte Menge zu seinen Scheidensekrotuntersuchungeii aus-nahmslos Co elio t omirte Frauen, etwa am IG. Tage nach der Operation, weil hier zufallige Verunreinigung der 
Scheide durch Berührung von aussen mit Sicherheit auszuschliessen war. Diese eine Versuchsanordnung ist allerdings von fundamentaler $\Lambda$ Vichtigkeit, da sie allein die Gewähr giebt, durch Zufãlligkeit nicht beeinträchtigt zu werden. Die zweite Abänderung gegen sonst übliche Methoden bestand darin, dass Menge kein Röhren-speculum die Vagina einführte, um die Sekretentnahme auszuführen, sondern mit einem verschliessbaren Löffel unter Auseinanderhalten der Labien in den Scheidengrund einging und hier eine Sekretprobe entnahm. Die Möglichkeit, Sekret aus dem Schcideneingarig nach den hinteren Theilen der Vagina zu verschleppen, wird hierdurch bedeutend herabgesetzt. Unter 70 auf diese Weise ausgeführten Versuchen blieben 62 Mai die Agarplatten absolut steril! Welcher Unterschied zwischen dem Scheideneingang und dem Scheidengrund hesteht, gelit am besten daraus hervor, dass von den vom Scheideneingangs-sekret angelegten Culturen G6 Mai Bacterienkolonien auskeimten und nur 4 steril blieben. Unter den in den 8 Fallen angegangenen Culturen (aus dem Scheidengrundsekret) liess sich in 7 Fallen nachweisen, dass die rein-gezüchteten Colonien keine pathogene Bacterien waren. Da stets ehen-dieselben Bacterien auch aus dem zugehörigen Scheideneingangssekret - nur in sehr viel grösserer Zahl - r, uchsen, so spricht Verf. mit Recht den Verdacht aus, dass trotz grosser Vorsicht, sowie Hineinführen ties Löffels Bacterien nach hinten verschleppt wortlen waren. Nur in einem einzigen Falle (unter den 70 untersuchten Frauen) liess sich der Streptococcus pyo-genes nachweisen, hier allerdings in grosser líeichlichkeit.

Derselbe war bei zahlreichen Versuchen an derselben Fran stets - immer zusammen mit dem Gonococcus - zu finden, so dass hier an eine zufallige Verunreinigung nicht zu denken war. $\lambda$ Vie erwähnt, waren diese Versuche mit alkalischem Agar angestellt. Zur Controle hatte Verf. nun gleichzeitig in 12 Fallen auf saures Agar ge-impf't. Während unter diesen 12 Fallen vorher 3 mal Wachsthum entstanden war, wuchsen auf dem sauren Boden G mal Culturen (meist Stäbchenbakterien). Es haben aber diese Befunde nur ein untergeordnetes Interesse, weil sämmtliche pathogenen Bacterien ausnahmslos auf alkalischen Nährböden ge-

278

Buchanzeigeti.

deihen, so dass jene auf sauren Nährböden gewachsenen Keime bestimmt keine patliogenen Pilze sein können.

Dasselbe gilt von einer dritten Versuchsreihe, die er an eben jenen 12 Frauen mit hochgeschichtetem Traubenzucker-Agar vornahm. Hier gingen 11 mal Culturen an. Das fast ausnahmslose Vorhandensein von Anaëroben in der Vagina (die ebenfalls nicht pathogen sind) ist hiermit erwiesen.

Aus dem fast ausnahmslosen Freisein der Vagina von Infectionskeimen liess sich z $\Lambda$ var von vornherein der Scliluss zielien, dass die Vagina bak-tericide Eigenschaften haben müsse. Uni diese Vermuthung jedoch sicher zu erweisen, nanm $M$. in grossem Umfange Impfungen der Vagina selbst vor. Hierzu $\Lambda$ vähíte er 23 mal den Bacill. pyoceaneus (blanen Eiterpilz), 30 mal den Staphylveoccns aureus und $27 \mathrm{mal}$ den Streptococcus pyogenes. Alle diese Pilze. welche friscli gezüehtet waren und eine liolie Virulenz aufwiesen, wurden in Reinkultur mittelst eines Glasstabes in den Scheidengrund ein-geführt. Das höchst interessante Ergebniss dieser Versuche war, dass längstens nach $70 \mathrm{St}$ und en sicb die Sclieide dieser fremden Ein-dringlinge mit völliger Siclierhoit entledigt hatte. Ganz besonders wichtig war alter liierbei die gleichzeitige Feststelïung, dass das Vorhandensein von saurem, alkalischem oder amphoteren Vaginalschleim keine Ver-schiedenheit ausmachte, dass alle 3 Sekretarten gleich schnell und gleich sicher die Abtödtung der Pilze vollzogen. Verfasser stellte nun sehr uin-fangreiche $\Lambda$ Гersuche an, um der 
Ursache dieses (von ihin. Selbstr einig ung der Sclieide benannten) Processes auf die Spur zu kommen. Als bekannt darf wohl die Arbeit Doederleins vorausgesetzt werden, welcher den von ill in zuerst nachge $\Lambda$ viesenen (anaërob wachsenden) Scheidenbacillen diese Aufgabe zuertheilt. Nach Doederlein produciren die Scheidenbacillen Milchsäure; sie sind die Ursache der sauren Reaction des „normalen” Sekrets, und nur durch diese Säure machen sie die Vagina unbewohnbar für die (einen alkalischeii Boden verlangenden) patliogenen Bacterien. Indem wir wegen der D o e derle in'schen Begriffe „normales” und ..pathologisches4' Sekret auf das Referat der Krönig'schen Arbeit verweisen, seien hier nur die obigen Impfversuche Menge's noch einmal hervorgehoben, durch die er unzweifelhaft feststellte, dass auch den alkalischen Sekreten die gleiche Fähigkeit zur Abtödtung pathogener Pilze innewohnt wie den sauren. Nach der Ansicht Menge's spielt die von den ,,Scheidenbacillen” ge-lieferte Milchsäure zwar eine gewisse. aber doch nur untergeordnete Rollo in dem Selbstreinigungsprocess. Eine etwas grössere Rolle kommt der ursprünglich (bei Neugeborenen bei Freisein von Scheidenbacillen) schon vorhandenen Säure zu. Ein weitcres Moment ist die lebhafte Leucocythose und Phagocytose, die Verf. nach Hineinbringen von Staphylokokken in die Vagina direkt mikroskopisch nachweisen konnte. Die Hauptaufgabe an diesem Veruichtungsprocess fällt aber den die Sclieide bewo linen den Anaëroben und ihren S t o f f we c hselpr o d net en zu.

Zum Beweise dieser Behauptung fiihrt M. folgende Versuche an: Er entnahm saures Sekret aus der Vagina, brachte es in verschiedene sterile (vor Eintrocknung geschützte) Behälter und impfte dasselbe mit Staphylokokken. Die Abtödtung der letzteren erfolgte jetzt ebenso rasch, wie ini Thierkörper. $\Lambda$ Venn er jedocli die Sekretprobe vorher durch strömenden Dampf sterilisirte, so hielten sich die Staphylokokken sehr viel längere Zeit oder starben überhaupt nicht ab (trotz des sauren Sekretes). Machto er die Sekretprobe durch Sodazusatz schwach alkalisch, so trat der Tod der eingeimpften Staphylokokken etwas verlangsamt ein. Sterilisirte er das alkalisirte Sekret durch strömenden Dampf, so bildete es für nun aufgeimpfte Staphylokokken den vorziiglichsten Nährboden! Diese Er-gebnisse sind so eindeutig, dass sie in der That überzeugend $\Lambda$ virken. Da auch die Sclieide Neugeborener, wie M. nachwies, die Fähigkeit der Selbstreinigung besitzt, so schliesst er, dass der Gewebssaft an sich und der Sauerstoffmangel weitere Faktoren sind, durch die die Vagina sich von patliogenen Pilzen frei zu halten vermag.

Es giebt nun auf der anderen Seite eine grosse Reihe von Faktoren, welche diese der Scheide innewohnende Kraft herabzumindern und tinter

Buchanzeigeni

279

Umständen sogar ganz aufzuheben vermügen. Als solche sind in erster Linie die Menstruation zu nennen, welche das Vaginalsekret alkalisirt, natür-liche Scheidenbewohner zum Theil herausschwemmt, vor Allem aber das Sekret so verdünnt, dass es seine gewöhnliche Schutzkraft verliort. Ferner starke Sekretion aus dem Uterus, besonders bei gonorrhoischer Infection desselben. Auf die Bezugnahme M e n g e 's auf die Verhältnisse der sehwangeren Scheide gelien wir an dieser Stelle nicht ein.

Die Möglichkeit, dass in der Vagina Niclitschwangerer sich pathogene Keime befiuden, giebt er also z $\pi$ : es sind dies alter stets Ausnahmen, als solche erkennbar und wohl auch meist durch bestimmte Verhältnisse er-klärbar.

In dem einen Falle (unter 10 Fallen), in welch em Menge reichliche Streptokokken im Scheidensekret (bei gleichzeítiger Uterusgonorrhoe) ge-funden hatte, stellte er Versuche an, die Scheide durcli Lysol, Garbol, Sul > li-mat, Milchsäurelösung in versehiedener Concentration und verschiedenen Zwischenräumen zu desinficiren. Das Resultat war, dass nach 3-( > Stunden sich 
stets wieder reichliche Streptokokken aus dem Sekret züchten liessen! Dagegen liess eine zweimalige Aetzung des Uterusinnern mit 50 procent. Chlorzink die Staphylokokken aus dem Vaginalsekret verschwinden.

Das nächste Capitel handelt von den Bacterien des gesunden und kranken Uterus und (Kir Cervix. Verf. konimt liier zu folgenden Schlüssen: Der äussere Muttermund bildet bei dem sehwangeren und nichtschwangeren Weibe unter gewöhnlichen Verhältnissen die Grenze zwischen bakterien-haltigem und bakterienfreiem Abschnitte des G-enitalkanals. Weder im Sekrete livch im Scheidengewebe der normalen Uterushöble (Corpus und Cervix) leben Bacterien, welche auf unseren gebräuchlichen (alkalischen) Nährböden gedeihen. $\Lambda$ Veder im Sekrete noch im Schleimhautgewebe soldier Uteri, welche im Corpus oder in den Cervix oder in beiden die anatomischen Merkmale fur die einzelnen Formen der sogenannten chronischen Endo-metritis zeigen, leben Bacterien, welche auf unseren gebräuchlichen Nährböden gedeihen, ausgenommen der Gonococcus und der Tuberkelbacillus.

Die Untersuchung der Cervix nahm Menge, im Gegensatz zu anderen Untersuchern, von oben vor, unter Aufschneidung des Uterus. Seine Re-sultate sind dadurch sehr $\Lambda$ riel zuverlässiger, weil er ein Nachoben-verschleppen von Keimen mit Sicherheit ausschliessen konnte.

Die Keimfreiheit und die Schutzkraft der Cervix ist nach seiner An-sicht eine so absolut sichere, dass er das Ausbrennen ties Cervikalkanals nach supravaginaler Amputation des Uterus nicht nur als unnöthig, sondorn direkt als schädlich verwirft, weil eben die Schutzkraft des Epithets darunter leidet. $\mathrm{De}^{\wedge}$ Ferneren meint er, dass die von A. Martin eingeführte Totalexstirpation des myomatösen Uterus per Coeliotomiam von einer vüllig un-richtigen Voraussetzung ausgehe. Martin sehe $>$ in dem zurückbleibendeu Cervixstumpf eine gefährliche Infectionsmügliehkeit und in der direkten Communikation der unterbundenen Ligament-Stiimpfe mit der Scheide das beste Mittel, eine Infection zu verhüten. Gerade das Gegentheil sei richtig, da vom normalen Cervikalkanal niemals eine Infectionsgefahr droht, und da durch die küustliche Communication zwischen dem Peritoneum und der nicht immer aseptischen Scheide eine aufsteigende Infection ermöglicht wird, Wenn wir auch nicht glauben, dass der Streit zwischen der Totalexstirpation und supravaginalen Amputation durcli solche theoretische Erwägungen allein entschieden $\Lambda$ verden wird, dass vielmehr die Erfolge und die persön-lichen Erfahrungen der einzelnen Operateure die endgültige Entscheidung allmählich fallen wird, so sind diese bakteriellen Gesichtspunkte doch gewiss beherzigenswerth.

Indem wir hinsichtlicli der gonorrhoischen Erkrankung des Uterus auf die Originalarbeit verweisen, sei hier nur erwähnt, dass die chronische Endometritis nicht bakteriellen Ursprungs ist. Die baktericiden Eigen-schaften der Cervix schätzt Menge ausserordentlich hoch. Diesen Nach-weis erbrachte er durch Verinipfung von Staphylokokken und Streptokokken in die Cervix bei lõ Frauen. Nach längstens 12 Stunden war der Cervix-sclileim wieder steril.

280 Buchanzeigen.

Wenn in der Uteruskörperhöhle toter Nährboden in Gestalt von ge-stautem Sekret, von nekrotischen Neubildungsmassen, von retinirten Eiresten sich gehäuft findet, oder wenn die Uteruskörperschleimhaut wund ist, kann es ini Cavum eorporis für längere Zeit zu einer Bakterienvegetation konimen, vorausgesetzt, dass erstens bei physiologisch fuñktionirendem Cervixkanal artefieiell facultativ anaerobe Mikroorganismen mit Ueberspringung des Cervikalkanals direkt bis in den toten Nährboden oder bis auf die w-unde Schleimhaut hinaufgetragen werdeu, oder dass z weitens die Schutzwirkung des Cervikalkanales aufgehoben ist, sei es durch eine Carcinomentwíckelung im Endometrium cervicis, sei es durch eine Atrophie der Cervix-mucosa im höheren Alter oder durch eine Entfaltung des Kanals infolge des 
Tiefer-tretens von Neubildungen oder Eitheilen, welche einen direkten Bacterien-verkehi* zwischen Scheide und Uteruskörperhöhle vermitteln.

Das letzte grössere Kapitel behandelt die Bacterien der Tube und (.) varien.

Die gesunde Tube untersucht Menge 83 Mai (von 50 Frauen stammend). Nur in einem einzigen Falle liessen sich Bakterien nach $\lambda$ veisen! Es bestand hier gleichzeitig eine Wundinfektionsendometritis bei Collum-Carcinom. Sunst erwies sich die gesunde Tube stets steril.

Hydrosalpinx wurde "20 nial und Haematosalpinx 3 mal im Ganzen in '218 Aussaaten untersucht. Wiewohl auch auf Gonokokken und Tuberkelbacillen die Untersuchung ausgedehnt wurde. war das Ergebniss ausnahmslos ein negatives.

Am umfangreichsten waren naturgemäss die Untersuchungen der Pyosalpinx und der Salpingitis purulenta, da die Frage, ob der bei der Adnexoperation in die Bauchhöhle leicht hineingelangende Eiter infectiös ist oder nicht, praktisch von der allergrössten Bedeutung ist. Schon vor einer Reihe von Jahren hatte Menge über diesen Gegen-stand eine Untersuchung, die sich auf 26 Fälle bezog, verúffentlicht. Er hatte damals dreimal Gonokokken, zweima] don Streptococcus und einmal den Staphylococcus albus, ferner einmal einen Anaërob enund einmal einen Saprophyten gefunden. Zu diesen Untersuchungen hat er jetzt 96 neue ge-fügt; im Ganzen waren 122 Fälle mit 1726 Aussaaten verarbeitet worden.

In 75 Fallen erwiesen sich die Tuben steril. Unter den 47 Fallen, in denen Bacterien gefunden wurden, fanden sich

Gonokokken 28 mal

Tuberkelbacillen 9 ,

Streptokokken $3 \quad$,

Staphylokokken 1 ,

Bacterium coli com. 1

Saphrophyten 1 ,

Anaerobe Bact. 1

Bacteriengemische 3 ,

(darunter einmal mit Streptokokken)

An dieser Tabelle ist besonders interessant, wie selten sich Staphylo-und Streptokokken im Tubenoiter nachweisen liessen, im Ganzen nämlich nur fünfmal. Und zwar schrumpft diese Zahl noch melir zusammen, wenn man die letzten 96 Fälle für sich betrachtet, bei denen Menge eine andere (also voraussichtlich bessere) Methode der Sekretentnahme eingeschlagen hat. Unter diesen 96 Fallen finden sich nur zwei Befunde von Streptokokken, kein einziger von Staphylokokken.

Referent erblickt in dieser Feststellung eine sehr willkoinmene Be-stätigung seiner eigenen Untersuchungen, die er seinerzeit an 10 Pyosalpinx-fällen angestellt hat, ohne auch nur einmal Staphylo- oder Streptokokken gefunden zu haben. Die Richtigkeit der Untersucluingsresultate von Boisleux und Witte, welche beide im Pyosalpinxeiter eine Mikroflora gefunden haben, dereu „Mannigfaltigkeit in der That, nichts zu wünschen übrig lässt,” be-zweifelt Menge dalier entschieden.

Unter acht Fallen von echtem 0 varial absce ss wurde dreimal der Gonococcus, zweimal Tuberkelbacillen ami einmal der Streptococcus nach-gewiesen, drei Fälle hatten sterilen Inhalt. Unter 18 Fallen von Pseudv-

I $>$ uehanzeigen. $\quad 281$

abscessen ini Ovariuiu land sicli sechsmal dor Gonocoecus: zW $<$ “ $>$ IГinaI war dor 1·́ífer bakterien†'reï. 
Das Peritoneum wurde 32mal baktcriologiscb imtersucht. Es wurde $\mathrm{I} \Gamma>$ mal dor

Tuberkelbacillus, achtmal d.jr Streptococcus (darunter $1>$ efanden sich fünf Fälle von

Operationsperitonitis) zweimal Gonokokken, zweimal Bacterium coli gefunden. Viermal war die Untersuchung ergebnis,slos.

Worm der Referent sicli aucli bewusst ist, zahlreiche iu†eressante (Jntersuchimgsreihen und Beobaehtungen in der Arbeit $\left\{\mathbf{-} /\right.$. B. über das Ver-lialten des Gonocoecus $\prod$ nd des Tuberkelbacillus) kaum gostreift zu haben? so hofft oi' doch, dass seine kur/.en Mittb.eilu.ngen dazu beitragen werden. zum Studium dieses $\backslash$ erkes aiizurogen, welches für ieden Gyuäkologen, der t'iir Bacteriologie Interesse besitzt odor wenigstens darauf VVertl·i legt, dass seine practischen Maassnahmen tin Einklang niit der theoretíschen $\mathrm{W}^{1} / 2$ sen-schaft stehen, fortan kauni entbebrlicli seiu dürfte.

Fí'ir die lioffentlicb bald erfolgende zweite Autlage erlauben wir uns.

dem Verfasser zu empfehlen < nocli mehr Ünter-Kapitel einzuführen, und die

gerade zur Untersuchung stebenden Kragen und ibre Ergebnisse durcb den

i truck nocli melii · liervorzuheben. uni ein leichteres $\Lambda$ uf †ंinden einer Stelle

zn ermöglichen. R. Schaeffer-Berlin.

Die Beziehungen zwisehen Nase und weibliehen Gesehleehtsorganen

von Dr. IV. Fliess. Leipzig und Wien. 1897. Franz Douticke.

Der Verfasscr dor vorliegenden Schrift hat das Problem der Beziehungen zwisehen Nase und weibliehen Gesehleehtsorganen von eineni eigenartigen Gesíchtspunkte aus untersucht und ist dabei zu interessanten Resultaten gekommen, welche ltier zunächst referirend in Kürze mitgetheilt sein mögen.

Die unteren Muscheln und die Tubercula scpti siiid „nasale Genital-stellen”, welche während dor Menstruation anschwellen und enipfindlich werden und deren pathologische Beschaffenheit „nasale” Dysmenorrhoe be-dingt. Cocainisirung dieser Genitalstellen mit einer 20\%

Coeainlösung be-seitigt die dysnienorrhoischen Beschwerden nionientan, Aetzung der Stellen. mittels Trichloressigsäure vermag die Dysmenorrhoe dauerncl fern zu halten. Dieselbe ist aucli oft bei Entbindungen vertreten in Form des, .echten Wehenschmerzes" und kann ebenfalls durch den Cocaiuversucli von tier Nase her ausgesehaltet werden. Aucli während der Schwanger.sc 'haft und während der Lactation giebt os menstruelle Yeränderungen in der Xaso. denn der Mons †ruationsprocess besteht während dieser Zeit fort, findet aber nicht soinen gewöhnlichen A.blauf durcb die uterine Blutung: während der Schwangersehaft speichern sich die nienstruellon Antriebe auf und führen normaler Weise nach 10 Menstruationsintervallen die grosse Menstruation, den EntbindungsvoГgang, herbei. Für den Abortus gelten die analogeii Yoraussetzungen wie für die normale Entbindung; or tritt zu nienstruellon Xeiten ein, hat Beziehungen zur Nase, von dor aus or künstlich hervorgo- $\pi$ if'en werden kann. In dor Menopause hört der Einfluss der nienstruellon Vorgänge auf die Nase nicht auf, an Stelle der Blutungen kõnnen alsdann, und zwar an don poriodisolieu ïagen, Migräne, Pulsarythmie u. dergl. troten, Beschwerden, $\Lambda$ velche sich ebenfalls oft durcb Behandlung der nasalon Genitalstellen beein†lusse $\pi$ lassen. Beim lícproductionsact bekunden sich die Beziehungen. zwisehen Nase und Sexualität offers durch Nasenbluten und Schnupfen inFolge des Coitus: bei Masturbanten zeigen die nasalon. Genitalstellen tvpische Yeränderungen. Fernerhin versueht dor Verfasser durcb.

Analysen von Schwangerschaftsbeobachtungen zu erweisen, dass der Men-struationspxocess während. der Schwangersebaft fortdauert, sowio dass die Schwangerschaftsbeschwerdon und die sie begleitenden nasalen Zeichen aus-schliesslich periodisch auftreten, und zwar in zwei Gruppen von Reihen: in it 28tägigen und 2,' > tägigen Intervallen. in diese Reihen fallen Milch- 
secretion, Kindsbewegungen, Yorwehen, vorzeitiger Fruclitwassorabgang und. dor Beginn des Kreissvorganges, nach dor Entbindung das Eintreten $\mathrm{d}^{\wedge} \mathrm{r}$ 1. und aller folgenden Menses. Das Nebeneinanderbesteben dor periodischeu Reihen mit 28- und 2,'itägígon Intorvallen orklärt aucli die TJnregelmässig-keiten der Menses, an deren Stelle oft Ersatzorseheinungen treten: Migräne. Monatsschrift für Geburtshülfe und Gynaekologie. Bd. V. Heft 3. 19

\section{Buchanzeigon.}

Angst- und Asthmaanfälle. Die periodischen Reihen wertlen von dem mütterlícheii Organismus auf das Kind iíbertragen und setzen sick, tlnreli das ganze Leben desselben fort, sie sind daher bei alien Kindern derselben Mutter aufzeighar und verknüpfen die Generationen untereinander. Die nienstruelle Blutung ist demnaoh nur ein verhältnissmässig uiiwesentliches Symptom, eines grossen Processes, der beiden Geschlechtern und jedem Alter eigen ist und von dessen Störungen das Eintreten vieler constitutioneller Krank-lieiten abkängt, das ferner das Eintreten und die Krisen $\tau$ on gewissen Infectionskranklieiten bei vorhandener baeterieller Ursache bestimmt und dem endlicl) die wichtigen Vorgänge ties Auf- und Abbaus unseres Körpers, einsehliesslich tier Geburt und ties Todes, unterliegen. Audi tlie Frage nach der Entstehung des Geschlechtes, den grösseren Knabenüberschuss und den schliesslichen $\Lambda$ usgleich des Gesclileclitsverliältnisses bringt der Verfasser in it dem Besteken einer 28-tägigen wo iblichen und einer 23 tägigen in a $n$ $\mathrm{n}$ I i e h en Periode in Yerbindung: demnach hänge das Geschlecht des Kinder davon ah. eine wie geartete Periode (mi'innliclie oder $\Lambda$ veibliche) das befrnclitete Ei zuorst nach der (.'(inception treffe.

Der originellste Theil der in†eressanten Sclirift ist der Yorsuclt, die koitlicli unverrüekbare Gesetzmässigke-it einer in der Sexualität wurzelnden Periodicität ini Aufbau und $\Lambda$ bbau des menscblicheii Körpers nachzuweisen. Zeifellos ist die Periodicität a.1 ler.Lebonsorscheinungen eines tier interessantesteu und wichtigsten biologischen Probleme. Der Verfasser begründet seine Aus-führungen ini wesentlichen nur mit der Zusammenstellung und Reihenbildung kritiseher Tage in einigen Ivrankongeschichten, in denen er die genannten $2<$ Stägigen und 23tägigen Perioden zn erkcnnen glaubt. Er verspriclit zuiu Schluss die Frage der Bedeutung der Periodicität beiMenscben, Thieren und Pflanzen ausfülirlicli monograpliisch $\kappa u$ bearbeiten, vielleicbt bringt er dann $\Lambda$ vissenscliaftlicl] ljesser begründete Beweise seiner Itleen als in der vor-liegenden Sclirift. $\Lambda \backslash$ as das eigentliclie Thema des Buches anbelangt. nämh'ch die Bezielmngen der ẃeíbliehen Sexualorgane zur Nase, so be-s†ehen dieselben nach Ansiclit ties Verfassers in einer gegenseitígen reflek-†orischen Beeiiitlnssung. Es ist. hinlänglicb bekannt, wie sebr die kritik-los $<\cdot \Lambda$ nwendiing der $\square$ in eingescliränkterer Form kaum anfeclitbaren - llael $<$ "schen Lelire von der nasalen Keflexneurose auf alle müglichen nervösen Störungen dem Fortscliri†† und dem $\Lambda$ nseben der jetzt so entwickelten ßlii-nologie geschadel hat. I'm so notliwentliger aber musste tier Verfasser, da er ini wesentlichen eine wei†ere Ausfübrung dieser Lelire bringt, sich. mit < $\mathrm{h} \cdot \mathrm{n}$ entgegengesetzten $\Lambda$ nscliauiingen antlerer $\Lambda \mathrm{u}$ †oren $\Lambda$ venigstens abfinden, während er die Litteratur seines Themas nur so $\Lambda$ veit heranzieht, als sie seinem Gedankengange entspricht. Selbst wenn wir annelimen, dass tlie lnitgetheilten Exporimente und Krankengeschicliten exakt beobachtet sind. so beurtlieilt ador $\Lambda$ ul or den Erfolg der Cocainisirung und Aetzimg der unteren Nasen-musclieln und der Tubercula scp†i z. B. bei Dysmenorrboe docli sicker etwas einseitig. denn die $\mathrm{P}>$ edoufung, welcke für den gelegontlichen Erfolg des Experiinentes et $\Lambda v a$ der Suggestion, eventuell der Hysterie und ferner dem narkotischen Einfluss des Cocains zukonimen dürl†,e. wirtl nickt kritisch untersuckt. aucli wie eiiientlicli tlurcli tlie Oocainisirimg eine vorübergehentle und dureb die Mortificirung eines Tkeiles der Nasenschleimhaut eine dau$\mathrm{crn}<$ le Einwirkung auf die Menstruationsbesckwerden zu S † tantle $1<$ vmmt -was docb bei der 
ganzen. Saclie das $\lambda$ Vich $\dagger$ igste ware - wirtl in keiner $\lambda$ Yeiso zn erklären ,versuc $\cdot$ li†: tier Loser bleibt lediglich angewiesen auf das Scklagwort „,r efl ekt oriscli “, bei dem er sich nicbt allzuviel vorztistellen vermag. - Jedenfalls alter ist das Buck anregend und interessant geschrieben: stdton aus diesem Grunde kann es zur Lektüre empfohlen werden.

B o t † e r m u n tl - Dresden.

Genaue anatomiseh-histologisehe Untersuchuiig • von zwölf Ovarien-tumoren von Friedrích Vogl. München 1896. Th. Ackermann.

Yerf. gieltt eine Beschreibung der makroskopischen und mikroskopiscken Befunde von 12 ini Verlauf eines Semester in der Münchener Frauenklinik < '.xsi irpierten Ovarialtumorcn und /.war von 1() cvstisehen und 2 soliden.

Tagesnaehrichten und Notizen. 283

Unter don cystisclien ist besonders interessant oin noch sehr kleines uni-loculä $\Gamma \theta \mathrm{s}$ proliferierendes Cystoma glandulare (No. 2) ferner eine proliferierende $\mathbf{c}$ Cyste mit pseudomyxomatösen Veränderungen am Peritoneum des Lig. lat. (No. 3, leider fehlen Mer Angaben über die Beschaffenheit des Cysteninhalts.) Ks reilien sicli an 2 carcinomatös degenerierte glandulare Cysten, von denen eine (No. õ) Fliminerepithel trug, eiu Cystocarcinom, drei multiloculäre glandulare Cystome, von denen eines (No. 8) gallertigen Inhalt zeigte und .sehliesslich noch ein Flimmerepithelcystom des Ovarium.

.Die beiden soliden Tumoren waren Sarcome, das cine ein Endo-1/8helioma lymphaticum, das zweite ein Endothelioma peri-vasculare.

$\Lambda \mathrm{V}$ e s t $\mathrm{ph}$ a 1 e $\mathrm{n}-$ Flensburg.

Tagesnachrichten und Notizen.

Der Unfug der .,Lösuug des Zungenbäu dehens” (lurch Jlebanimeii 1st in Deutschland noch weit verbreitet. Daher sei ein in der „Lancet” (vom “23. Jan. 1897) berichteter Fall von. Verblutung im Anschluss an diese „Operation” auch hier niitgetheilt. Gleich nachdem die lleban $\pi$ ne das Frenulum linguae mittels Scheere durchsclmitten hatte, soil es nicht geblutet haben, aber am folgenden Tage rann schubweise Blut aus dem Munde, den sie dann mit Wasser auswusch. Erst nach weiteren 24 Stunden wurde ein. Arzt geholt, der das Kind in ein Krankenhaus schaficon liess, wo es ein paar Stunden nach der Aufnahme stafb. Die Zmigenwundo hatte eine Lä,nge von $\mathrm{P} / \mathrm{i} \mathrm{cm}$. Magen und Därme enthielten sehr viel Blut.

1st schon Siujl und Zwcck der Durchschneidung des Zungenbändchens ein sehr problematischer. so sollte diese ..Operation", wenn von Hebammen ausgefiihrt, strong geahndet werdeu.

Der diesjährige Congress í'ür innere Mediein” wird in. Berlin. zı gleicher Zeit abgehalten $\Lambda$ verden wie der Deutsche Gynäkologen-Congress in Leipzig, in der Pfíngstwoche vom if.-12. Juni. Verhandlungsthemata sind: Die Behandlung des chronischen Gelenkrheumatismus, Epilepsie und. Morbus Basedo $\lambda$ vii. Von Vorträgen, $\Lambda$ velclie auch lïlr den Gynäkologen von. Interesse waxen, sind Iris jetzt angemeldet: A. Fränkel und C. Benda-Klinische und anatomische Mittheilimgen über acute Lcucämie; C. Lieb-rei.ch: die Ziele der inodernen medicamentösen Therapie; M. Mendelssohn: Die klinische Bedeutung der Diurese und die Hilfsmittel ihrer thera: peutischen Beeinflussung; A. Baginsky: Zur Pathologie und Pathogenese der kindlichen Somnierdíarrhöen.

Personalien.

Privatdocent Dr. L. Kessler in Dorpat ist zum ausserordentlicheii

Professor era. ami t $\Lambda$ vorden. «

Dr. F. Leocata hat sich als Privatdocent fur Geburtshülfe und Gynä-kologie an der Universität Neapel habilitirt-. 
I $93 / 8$ 